\title{
Localization and loss of coherence in molecular double-slit experiments
}

\author{
BJÖRN ZIMMERMANN1,5, DANIEL ROLLES2,6, BURKHARD LANGER3,7, RAINER HENTGES2, MARKUS BRAUNE2, \\ SLOBODAN CVEJANOVIĆ2,8, OLIVER GESSNER2,9, FRANZ HEISER2,10, SANJA KORICA2, TORALF LISCHKE2,11, \\ AXEL REINKÖSTER², JENS VIEFHAUS2,12, REINHARD DÖRNER ${ }^{4}$, VINCENT MCKOY' AND UWE BECKER2* \\ 1California Institute of Technology, Pasadena, CA 91125, USA \\ 2Fritz-Haber-Institut der Max-Planck-Gesellschaft, 14195 Berlin, Germany \\ 3Max-Born-Institut für Nichtlineare Optik und Kurzzeitspektroskopie, 12489 Berlin, Germany \\ 4Institut für Kernphysik, Universität Frankfurt, 60486 Frankfurt, Germany \\ 5Department of Physics Tulane University, LA 70118,New Orleans, USA \\ 6University of Western Michigan, Kalamazoo, Michigan, USA \\ 7Physikalische und Theoretische Chemie, Institut für Chemie, Freie Universität Berlin, 14195 Berlin, Germany \\ 8Medical Faculty, Physics Department, University of Rijeka, 51000 Rijeka, Croatia \\ ${ }^{9}$ Chemical Sciences Division, Lawrence Berkeley National Laboratory, Berkeley CA 94720, USA \\ ${ }^{10}$ Ericsson AB, Torshamnsgatan 39 A, 16493 Kista, Sweden \\ 11Max-Planck-Institut für Plasmaphysik, 85748 Garching, Germany \\ 12Deutsches Elektronen-Synchrotron DESY, 22603 Hamburg , Germany \\ *e-mail: becker_u@fhi-berlin.mpg.de
}

In molecular double-slit experiments, the interference between emitted core electrons of diatomic molecules gives rise to oscillations in the observed electron intensity. Here, we explore this behaviour for photoelectrons emitted from $\mathrm{CO}$ and $\mathrm{N}_{2}$ by soft $\mathrm{X}$-ray ionization in the molecular frame, and we argue that in addition to the undisturbed emission process intramolecular scattering can lead to electron interference between the scattered and unscattered wave in two ways: two-centre interference between two spatially coherent emitters and one-centre self-interference. The latter is the signature of a loss of spatial coherence. The spatial scale over which the transition from two-centre to one-centre coherence occurs is the de Broglie wavelength of the scattered photoelectron in units of the bond length. These results highlight the fact that the molecular double slit is based on two independent uncertainty principles, $\Delta p_{x} \Delta x$ and $\Delta E \Delta t$, the second of which causes ongoing tunnelling between the two centres, even after the collapse of the electron wavefunction in real space.

\section{UNCERTAINTY AND SYMMETRY BASED COHERENCE}

Quantum coherence, a property of matter that separates the classical from the quantum world, is most clearly demonstrated in a double-slit experiment with single electrons ${ }^{1}$. It is based on the uncertainty principle between momentum and position and, if one takes the vacuum into account, it requires entanglement between complementary variables such as momentum and position or angular momentum components. For example, decoherence theory assumes that such coherence never disappears but is always transferred to other systems; in most cases it is transferred to a complex environment, where the individual phase information is lost and the system thus becomes classical ${ }^{2,3}$. In general terms, decoherence is caused by the entanglement of controllable degrees of freedom with other quantum states to encode "which way" information ${ }^{4-9}$. In the simplest case this could be a momentum kick where, assuming an infinite universe, the original phase information is, however, irreversibly lost. In addition, a system of two indistinguishable, and hence inversion-symmetric emitters such as a homonuclear diatomic molecule, where each electron has an equal probability of being located at and hence of being emitted from either atomic site, is also a source of spatial coherence ${ }^{10}$.
Experiments probing the quantum nature of matter by centreof mass interference deal with wave-front or amplitude-splitter based coherence imposed on single quantum objects via the uncertainty principle between momentum and position. The quintessence of these Young-type double-slit experiments is the wavefront splitting of a delocalized matter wave generated by beams of quantum objects with a momentum defined to better than the reciprocal of the corresponding uncertainty in position in units of the slit separation, and superposition of the two new waves. This coherent two-centre superposition is diminished by any observation of the quantum object that may result in a momentum change and hence an uncertainty larger than the reciprocal slit separation. Such interactions reduce the interference contrast and eventually provide "which way" information ${ }^{11}$. This is the result of coherence transfer from real to momentum space because the latter coherence defines the position as either "left or right" in real space.

In contrast, symmetry-based coherence is the result of electron tunnelling ${ }^{12}$ in an inversion symmetric molecule where the symmetry-induced energy splitting and corresponding tunnelling time are related through the generalized uncertainty principle between time and energy. Emission of the photoelectron from the indistinguishable left and right emitter sites is coherent but instan- 
taneous compared to the tunnelling time and hence randomly localized $^{13}$. It gains its time-dependent nonlocalization through the tunnelling process only. The electron wavefunction is split between two sites of a symmetric double-well potential and hence is in a nonlocalized state which is formally equivalent to a nonlocal, „,vacuum state-entangled“ one-electron state ${ }^{14}$. Viewed as a bipartite property of two real quantum objects ${ }^{15}$, entanglement is concerned with the quantum states of the combined photoelectron-ion system. Experiments employing this kind of coherence to probe the transition between quantum and classical behaviour are just evolving ${ }^{16,17}$.

\section{MOLECULAR DOUBLE-SLIT EXPERIMENTS}

At first glance, the molecular double slit seems to be simply a microscopic version of a wave-front splitter experiment. In both cases, interference is the result of coherence between two spatially-separated sites that give rise to a two-slit interference pattern. These interference fringes are the signature of phaselocked two-centre emission either in space, as in the famous double-slit experiment with single electrons ${ }^{1}$, or more recently in time $^{18,19}$. This is also the case in the molecule-frame photoelectron emission pattern of the gerade ( $g$ ) state of a diatomic homonuclear molecule such as $\mathrm{N}_{2}$, where two-centre interference causes a characteristic oscillation of the electron emission intensity along the molecular axis in momentum space. The ungerade $(u)$ state with its phase shift of $\pi$ between the two emitters also has an equivalent in macroscopic double-slit experiments either with a tilted incoming wave or, more recently, via entanglement of the slits through the polarization properties of the photons ${ }^{20}$. The result is a pattern of antifringes, where the dark and the bright stripes are interchanged $^{21}$. This corresponds to a parity change in the ungerade electron emission pattern of the molecular double-slit experiment with its phase shift of $\pi$ in the momentum space oscillation. We identified these oscillations by measuring the photoelectron diffraction intensities using angle-resolved high-resolution pho-

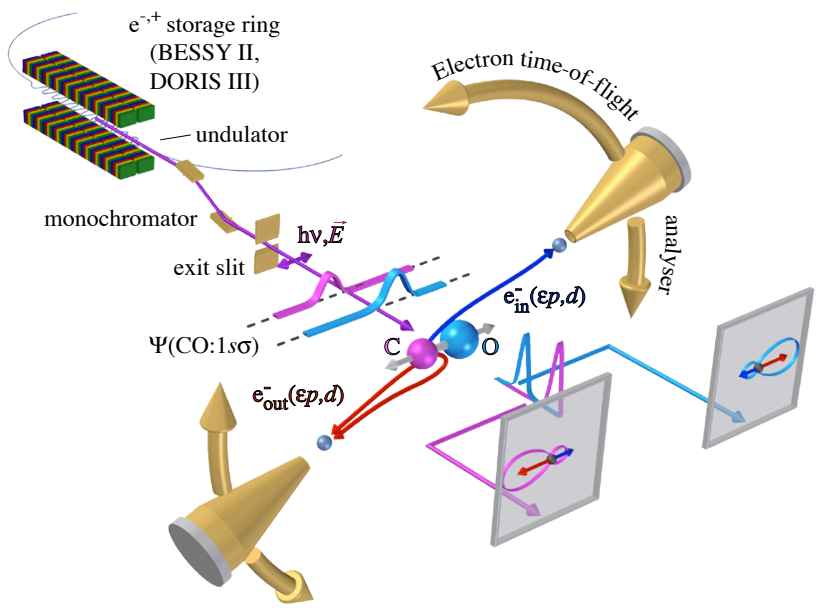

Figure 1 Schematic of the gas phase photoelectron diffraction experiment on CO. The experimental configuration chosen for recording the data reduces the experiment to a one-dimensional scattering experiment along the molecular axis and electric vector of the ionising radiation. The orientation of the molecular axis and the emission direction of the electron are determined by the coincidence of the photoelectron with the ionic fragments of the subsequently dissociating $\mathrm{CO}$ molecule. The photoelectron angular distributions in the molecular frame are shown for both emitter sites on their corresponding screens. The shape of the patterns is asymmetric peanut-like (data from Ref. 25) for both emitter sites ( $C$ and 0 ), but displays an inverted pattern structure for $C$ and 0 photoelectron lines, with respect to each other.

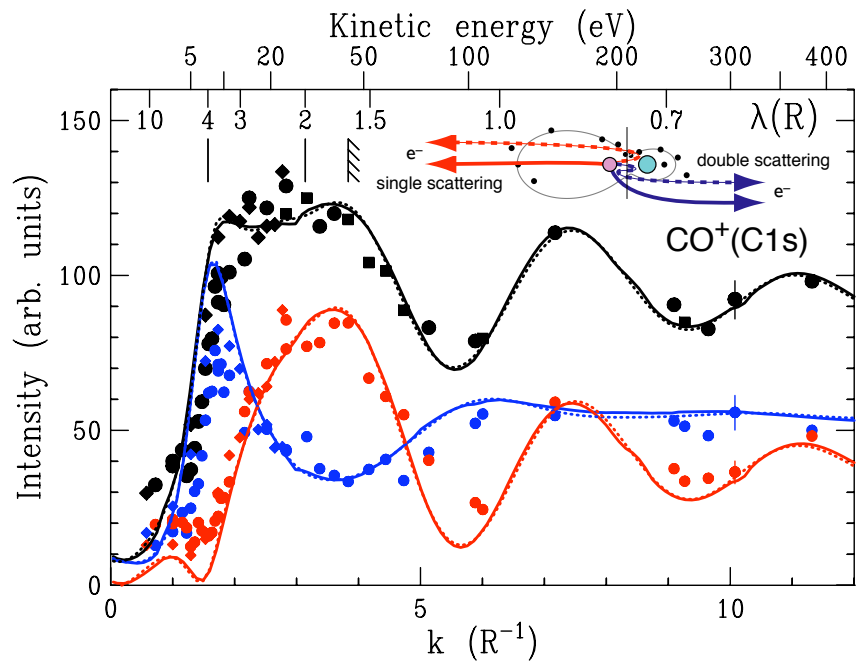

Figure 2 Photoelectron diffraction intensities versus electron momentum $\boldsymbol{k}$,

and de Broglie wavelength $\lambda$ (in units of the internuclear distance $R$ ) for $\mathrm{CO}$ molecules in the gas phase. Forward and backward scattering are differentiated by angle-resolved photoelectron - fragment ion coincidence. The red curves and data points represent the backward channel, the blue curves and data points the forward channel, and the black ones the sum of both. The solid lines are results of a partially relaxed-core Hartree-Fock (RCHF) calculation and the dotted lines are results of a semi-empirical one-centre interference model (see text). Our experimental results include data points taken at BESSY and at HASYLAB/ DESY (circles) as well as data from preliminary measurements at the Advanced Light Source (squares). The lozenges are reanalyzed data from Ref. 26. All diffraction intensities shown here are normalized to the corresponding partial cross sections to remove the exponential decay behaviour, which would otherwise mask the oscillatory structure. The experimental data and their fitting curve shown in the pictogram are from Ref. 25.

toelectron fragment-ion coincidence spectroscopy. Furthermore, we compared the case of core-electron photoemission in the homonuclear molecule $\mathrm{N}_{2}$ to a heteronuclear diatomic molecule such as $\mathrm{CO}$, which may be considered as a double-slit experiment with only one of the two slits open. In the photoemission experiment, the position of the open slit is selected by the choice of the appropriate kinetic energy of the emitted electron, i.e., either the $\mathrm{CO}$ : $\mathrm{C}(1 s)$ or $\mathrm{O}(1 s)$ photoemission-line from the carbon or oxygen site of the $\mathrm{CO}$ molecule, respectively.

At this point of comparison between the different double-slit experiments, the question of the limitations on the equivalence between molecular double-slit and macroscopic Young type double-slit experiments arises. Here we show that there are indeed distinct differences. Macroscopic wave-front splitting by a directionally fixed-in-space system of physically separated slits causes either pure spatial interference patterns or smeared-out slit projections, but does not include feedback between the two emission sites. The molecular double-slit experiment, however, differs in this respect. Here, photoelectron scattering or diffraction, a coherence effect in momentum space widely exploited in surface science $^{22,23}$, acts as an additional source of interfering waves and governs the angle-dependent photoelectron intensity in the molecule frame ${ }^{24-26}$.

To explore this intriguing feedback effect of interfering waves on the scattering, we performed photoelectron diffraction experiments on the isoelectronic hetero- and homonuclear molecules, $\mathrm{CO}$ and $\mathrm{N}_{2}$, in the gas phase. Fig. 1 shows a schematic of the experimental set-up for CO. It is an angle-resolved high-resolution electron-fragment ion coincidence experiment ${ }^{27}$ in which the electrons are detected by an array of time-of-flight spectrometers in a circle depicted by the solid arrows. The mass- and angle-resolved 
detection of the fragment ions is performed by a time-of-flight ion spectrometer with a position-sensitive anode, which makes it possible to determine the momentum of the fragment ions within a chosen acceptance angle of $\pm 25^{\circ}$ here. Measuring electrons and ions in coincidence in a gas phase experiment is equivalent to angle-resolved photoelectron spectrometry on free, oriented molecules. The analysis of the experiment hinges on the so-called axial recoil approximation, which assumes that fragmentation of the molecule occurs on a time scale much faster than rotation. The emitter atom can be selected by the kinetic energy of the photoelectron. The direction of its emission either into the molecule ( $\left.\mathrm{e}_{\mathrm{in}}\right)$, which is towards the neighbouring atom, or out of the molecule ( $\mathrm{e}_{\mathrm{out}}$ ), is determined by coincidence with an ion travelling either along with or counter-parallel to the electron. The two directions are also referred to as forward $\left(\mathrm{e}_{\text {in }}\right)$ and backward $\left(\mathrm{e}_{\text {out }}\right)$ scattering in the terminology of photoelectron diffraction. For both emission directions the scattered electron wave is superposed on the directly emitted electron wave, giving rise to pronounced oscillations in the emission intensities along the molecular axis, as shown in Fig. 2. The two forward ( $f$ ) and backward (b) states which define the emission direction and hence the emitter position are in the case of $\mathrm{CO}$ energetically non-degenerate and hence not entangled (for further details see the Supplementary Information).

Fig. 2 shows the results of calculations in the partiallyrelaxed-core Hartree-Fock approximation (RCHF) employing the Lippmann-Schwinger equation ${ }^{28,29}$ and the experimental data, as well as a fit of these data to a superposition of damped sine functions with continuous and discrete, bell-shape-like, damping curves (for details see methods). The first damping curve describes the extended X-ray absorption fine structure (EXAFS)type oscillations, whereas the latter represents the shape resonance components in the form of wave packets in momentum space representing quasibound high- $l$ states at multiples of the de Broglie wavelength $\lambda$. The agreement between theory and experiment is surprisingly good in view of the complexity of the problem. Both theory and experiment exhibit very pronounced oscillations in the backward channel, a fingerprint of a high degree of interference between emitted and scattered electron waves. In contrast, the oscillation in the forward channel is less pronounced because double scattering is much less probable than single scattering. The data also clearly show virtually identical oscillation frequencies in both channels, but with a phase shift of

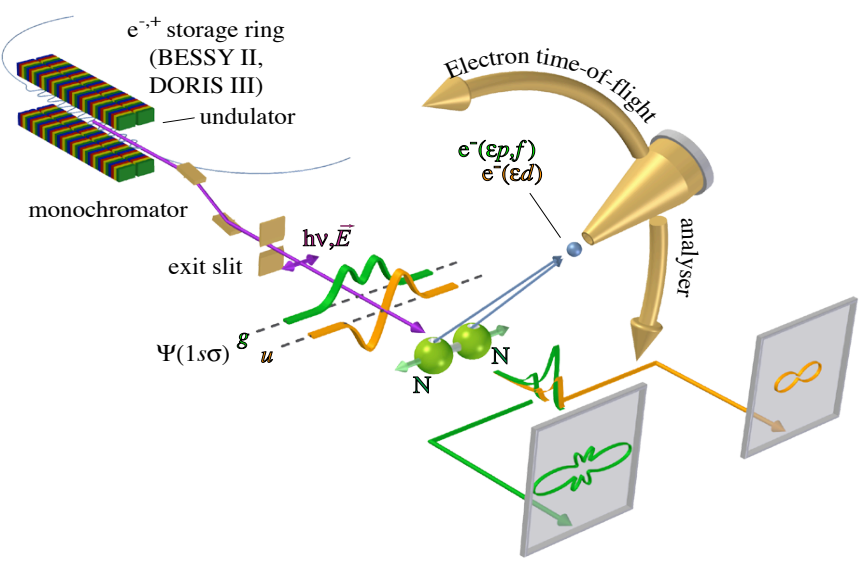

Figure 3 Schematic of the experimental setup for the high-resolution angleresolved photoelectron-fragment ion coincidence experiment on $\mathrm{N}_{2}$. The experimental set-up was similar to that for $\mathrm{CO}$ (Fig. 1) but with an emphasis on the energy-resolved detection of the gerade and ungerade states rather than on the directional separation of forward and backward scattering events. The moleculeframe photoelectron angular distributions for the gerade and ungerade state shown on the corresponding screens are taken from Ref. 16.

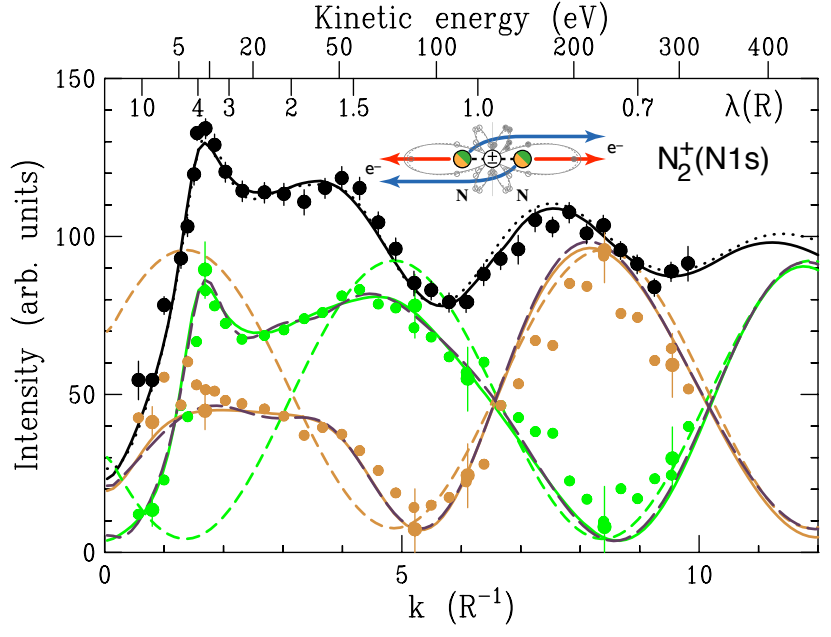

Figure 4 Photoelectron diffraction intensity versus electron kinetic energy for electron emission along the direction of the molecular axis in the homonuclear molecule $\mathrm{N}_{2}$. The horizontal axis notation is the same as in Fig. 2. The pictogram showing the superposition of the electron emitted from two separated spatial positions is displayed along with data from Ref. 16. The filled coloured circles are the experimental data for the gerade (green) and ungerade (yellow) state, and for the sum of both (black). Statistical and systematic errors are shown for the highresolution data points at low kinetic energies and the data points analyzed by two complementary methods. The latter error bars represent the bulk of the lowresolution data points. The non-scattered intensities shown by dashed lines are derived from the GCF model which simulates the photoelectron diffraction inten-

sities of the gerade and ungerade states by a superposition of two sine functions representing fractional intensities of the scattered (shown in Fig.5) and nonscattered electrons, respectively (see discussion in text). These simulated GCF intensities are shown as purple dashed lines for both symmetry states, the sum of them is presented as black dotted line. The normalization of the $\mathrm{N}_{2}$ diffraction data to the cross section was done in two ways, depending on the experimental resolution: For the high-resolution data below $k=4.7 R^{-1}$, the separate $\sigma_{g}$ - and $\sigma_{u}$-values were used, while for the data at all higher momentum values, $\left(\sigma_{g}+\sigma_{u}\right) / 2$ was used for both diffraction components.

$\pi$ between them because of the difference of one in the number of forward and backward scattering events (see schematic in the topright corner of Fig. 2). This distinction between the behaviour of odd/even scattering events corresponds to the slit distinction by left- and right-handed circularly polarized light in the macroscopic double-slit experiment of Walborn et al. ${ }^{20}$. The equivalence between the two experiments also holds for the case of homonuclear diatomic molecules where the two complementary superpositions, gerade and ungerade, corresponding to the fringes and antifringes in the Walborn experiment, are formed.

In analogy to Fig. 1 for the heteronuclear case, Fig. 3 schematically illustrates how the coherence properties in the homonuclear case were studied experimentally. The key instrumental issue in this case is the ability of the electron detector to resolve the gerade/ungerade splitting of the $\mathrm{N}_{2}{ }^{+}: \mathrm{N}(1 s)$ photoelectron $\operatorname{lines}^{30,31}$. Angle-resolved photoelectron - fragment ion measurements with high kinetic energy resolution of the photoelectron are very demanding and are at the limit of current instrumentation for the photon beam and particle detectors ${ }^{27}$. Our set of resolved data points supports the model of two spatially separated coherent emitters giving rise to distinct oscillations with a frequency inversely proportional to an apparent "single" bond length. The effective dynamical phase shift is $k r_{\mathrm{s}}$ with $r_{\mathrm{s}}=0.37 R$. The oscillatory behaviour of the sum of the $g$ and $u$ diffraction intensities of $\mathrm{N}_{2}$ is similar to the sum of the forward/backward contributions in the case of $\mathrm{CO}$. For observation at $0^{\circ}$ with respect to the polariza- 
tion vector and molecular axis, the frequency of this curve is proportional to "double" bond length. This behaviour, puzzling at first glance, is the result of intra-molecular scattering, i.e. photoelectron diffraction in $\mathrm{N}_{2}$. It exhibits spatial and momentum coherence corresponding to wave- and particle-like behaviour at the same time. To separate the intensity of the unscattered coherent electron emission from the scattered intensities, a sine function with a frequency proportional to the "single" bond length was fitted to the data and subsequently subtracted as described below. This sine function is referred to as $\mathrm{CF}$, because it is based on the Cohen-Fano model ${ }^{10,32,33}$ adapted to fixed-in-space molecules. In the following, we will refer to this combined one- and two-centre coherence model as the Generalized Cohen-Fano (GCF) model (see methods).

Fig 4 shows the fitted and extended sine curves $\mathrm{CF}_{\mathrm{g}, \mathrm{u}}$ of the GCF model and the calculated $g$ and $u$ diffraction intensities $\mathrm{RCHF}_{\mathrm{g}, \mathrm{u}}$ along with the sum of the $g$ and $u$ curves as dashed, solid and dotted lines respectively. The raw data for this fit were derived from the various RCHF curves as follows. For $k>7 R^{-1}$, $\mathrm{CF}_{\mathrm{g}, \mathrm{u}}$ is given by $\mathrm{RCHF}_{\mathrm{g}, \mathrm{u}}-$ EXAFS with EXAFS taken as half of the intensity oscillation of the $\mathrm{RCHF}_{\mathrm{g}, \mathrm{u}}$ sum curve. Subtraction of extrapolations of the fitted curves to low kinetic energies from the diffraction intensities yields the scattered intensity within the GCF

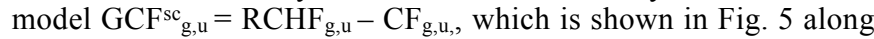
with representative data points for the different scattering regimes. Because of the ungerade character of the total wave function, the $f$-wave enhancement of the shape resonance essentially appears as an odd partial wave effect in the gerade channel only and complicates the analysis of the coherence behaviour. We therefore choose the ungerade channel (shown in yellow in panel a of Fig. 5) as a starting point of our analysis. In addition to the various fractional intensity curves, this figure shows schematics of the different twoand one-centre scattering processes of the GCF model (see methods). The corresponding analysis indicates that at the onset the scattered photoelectron intensity oscillates predominantly with a "single" bond length frequency (actually $0.63 R$ due to the same energy-dependent phase shift as for $\mathrm{CO}$ but with a potentialdependent dynamical phase-shift of $k r_{\mathrm{s}}$ with $r_{\mathrm{s}}=0.37 R$ ), which changes to a "double" bond length frequency at an energy around $k\left(R^{-1}\right)=2 \pi$. The "double" bond length frequency is the frequency exhibited by the sum of the intensity curves for the gerade and ungerade channels, where the separate intensities have been modulated with an amplitude of half of their sum but with no relative phase shift with respect to each other. In contrast to the $g-u$ state-specific modulation of the two-centre oscillations shown in panel a of Fig. 5, this final state behaviour shows no hint of the character of either symmetry state. The phase shift of $\pi$ of the scattered intensity with respect to the emitter intensity oscillations in the two-centre case gives rise to a decreasing modulation in the ungerade channel for $k$ values between 1 and 4 in units of the inverse bond lengths. This reflects the severe degradation of the Cohen-Fano emitter oscillation by an order of magnitude. In this regime, the de Broglie wavelength of the photoelectron is much larger than the bond length, and the spatial coherence between the two emitter sites is the dominating factor for both the scattered and unscattered electron to show wave-like behaviour. The $\pi$ shifted superposition of the two waves removes the primary quantum interference over several oscillation periods, thereby generating an unmodulated source of constant electron emission at the inversion centre of the molecule. In analogy to the standing wave regime ${ }^{34}$, where pronounced partial wave interference effects arise, the relevant wavelength scale for this behaviour is the bond length.

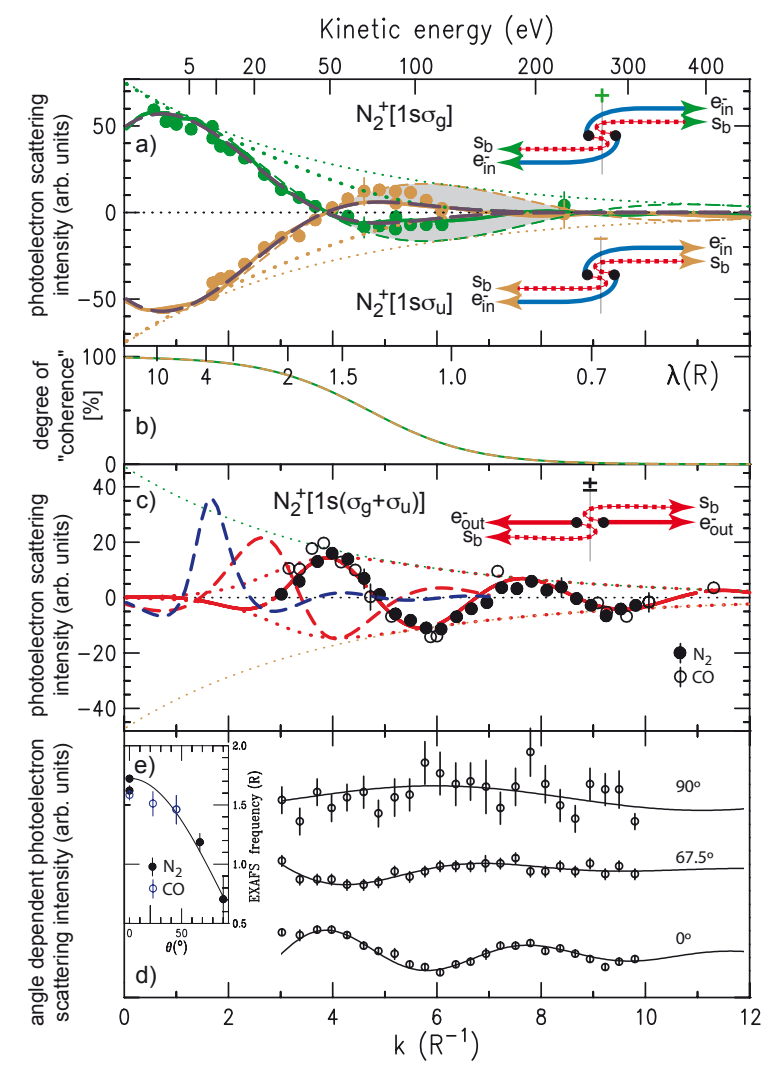

Figure 5 Scattering behaviour of the $\mathrm{N}_{2}$ ( $\mathrm{N}(1 \mathrm{~s}): \mathrm{g}, \boldsymbol{u}$ ) photoelectrons. a, Scattering behaviour for the $g$ (green curves and data points) and $u$ (yellow curves and data points) final ionic states. $\mathbf{b}$, The decoherence curve (described in the Methods section). Here, the direct emission intensity (CF curve), shown in Fig. 4 as a dashed line, has been subtracted from the corresponding total diffraction curve, shown in Fig. 4 by the solid lines. a, shows the two-centre oscillation as thin yellow $(u)$ and green $(g)$ and thick (purple for both $u$ and $g$ ) dashed curves for two different envelopes shown as thin and thick dotted lines. The thin dashed curves for $u$ and $g$ have a slope analogous to the one-centre curve in c, whereas the corresponding thick dashed curves are modified by the 'decoherenc' curve in b with the area between the two curves being shaded. The error bars of these data points are shown by a single representative bar. c, EXAFS-like one-centre oscillation (red solid line) with an envelope marked by a red dotted line. The filled black circles represent the sum of $g$ and $u$ scattered intensities. the open black circles show the corresponding datapoints of $\mathrm{CO}$ derived from the diffraction intensities of Fig.2. The red and blue dashed curves represent the fractional fitted intensities of the shape resonances in the standing-wave regime. The inset schmatic diagrams illustrate the different intramolecular scattering processes schematically. The solid and dashed lines with arrows represent interfering pathways of the photoelectron. d, Diffraction curves for the three different observation angles of the emitted photoelectron. Inset: The observation angle dependence of the oscillation frequency.

As the de Broglie wavelength $\lambda$ of the emitted electron becomes shorter than the bond length, the single-scattering process leads to a scattering-induced momentum change of the emitted electron larger than the inverse bond length and hence to particlelike localization at one atomic site, a process much faster than the tunnelling time. The resulting lack of overlap between the scattered electron wave and its neighbouring wave promotes their spatial localization and formation of a self-interference pattern, a behaviour closely related to the cancellation of geometrical phase effects in chemical reactions ${ }^{35}$. This momentum-dependent transition which transforms the coherent state into the non-coherent sub-tunnelling localized state is equivalent to that seen in 
environment-coupled decoherence experiments with their exponential loss of coherence with time and path separation. Excellent examples sharing the same physical footing as Heisenberg's uncertainty principle for position and momentum are photon scattering on coherent matter waves ${ }^{8}$ and the temperature-dependent wavelength of radiation emitted by heated fullerenes 9 . In contrast to other 'Gedanken' decoherence experiments ${ }^{36}$ that lead to left/ right asymmetries due to the gain of 'which way' information in a spatially fixed system, our transition, which is described by the same type of transition curve as shown in Fig. $5 \mathrm{~b}$, is a transition from two-centre spatial coherence to one-centre self-interference that gives rise to site-resolving, but random localization with no appearance of time-integrated left/right asymmetries. Instead, wave-like behaviour between scattered and unscattered electron emitted from two spatially separated points becomes particle-like scattering behaviour, resulting in position-resolving localization on the atomic scale in the sense of a Heisenberg microscope.

\section{ELECTRON ENTANGLEMENT}

At this stage more explicit definitions of the quantities involved may be helpful. Our system is one-dimensional and consists of two orthonormal basis sets, namely, the non-localized gerade $(g)$ and ungerade $(u)$ basis set of the parity states and the localized forward $(f)$ and backward $(b)$ basis set of the momentum states. These basis states are related to each other by

$$
\begin{array}{ll}
g=1 / \sqrt{2}(f+b), & \boldsymbol{u}=1 / \sqrt{2}(\boldsymbol{f}-\boldsymbol{b}) \\
\boldsymbol{f}=1 / \sqrt{2}(\boldsymbol{g}+\boldsymbol{u}), & \boldsymbol{b}=1 / \sqrt{2}(\boldsymbol{g}-\boldsymbol{u})
\end{array}
$$

The sum of the two components must be the same for both sets and hence unresolved data, either for energy or momentum, do not provide any information on our problem of localization and coherence in molecular double-slit experiments. The spatially coherent $g / u$ system (equation 1a) is transformed into the spatially non-coherent $f / b$ system (equation $1 b$ ) upon scattering. These $f$ and $b$ states, representing $n$ and $n$ - 1 collision events, respectively, show different $k$-dependent behaviour due to the relative phaseshift of $\pi$ between them as for the unscattered electrons, but with a different oscillation frequency. They are still superimposed forming $g$ and $u$ states but with a scattering-induced cancellation of their interference terms. In turn, for the case of the dominant backward amplitude, the probability of detecting a scattered electron in a certain direction is given by $b^{2}=1 / 2\left(g^{2}+u^{2}-2 g u\right)$, but here the interference term vanishes due to the orthogonality of the two non-degenerate basis states $g$ and $u$. The angular distribution of the scattered electron in the molecular frame is therefore still symmetric, but the phase coupling between the two separated emitter points is diminished by the pure appearance of the $f^{2}$ and $b^{2}$ amplitudes without the phase sensitive interference term $\pm 2 f b$. Hence this scattering- and symmetry- induced situation is associated with a loss of coherence in both basis sets $g / u$ and $f / b$, not anticipated by decoherence theory. The diminished interference term between $f$ and $b$ causes localization, but the vanishing interference term between $g$ and $u$ still preserves the symmetry, because their orthogonality is based on non-degeneracy in terms of the $g / u$ energy splitting $\Delta E_{g u}$ compared to the line width $\Gamma_{A}$ of the subsequent Auger decay, and not on the de Broglie wavelength dependent localization $\Delta x>\hbar /\left(2 \Delta p_{x}\right)$. The scattering channels $f_{s}$ and $b_{s}$ are equally strong in both of the corresponding symmetry channels $g_{s}$ and $u_{s}$ due to the relationship $\boldsymbol{b}_{\boldsymbol{s}}{ }^{2}+\boldsymbol{f}_{\boldsymbol{s}}{ }^{2}=\boldsymbol{g}_{\boldsymbol{s}}{ }^{2}+\boldsymbol{u}_{\boldsymbol{s}}{ }^{2}$. Therefore, there is no phase-specific element left in the two sym- metry channels for the scattered electron, a situation corresponding to depolarization in angular momentum space. Briefly, this is based on the fact that the parity eigenstates of two spatial positions which are the basis of equal probability for emission either from the left or from the right site are nonlocal ${ }^{14}$. These $g / u$ symmetry states become entangled to a singlet state built upon the joint photoelectron-ion system with its ungerade symmetry. This nonlocal symmetry state is now transformed into our non-coherent randomly localized scattering state that is characterized by phaseand hence parity-insensitive behaviour. The singlet state of the complementary momentum state $f / b$ system is the coherent localized state describing two anti-symmetrically oscillating electron emission patterns at the individual sites, but with mirror symmetry regarding the inversion centre of the two emitter sites. This state, caused by the degenerate part of the overlapping $g$ and $u$ states, will only be briefly mentioned here. The corresponding Bell-states have the form:

$$
\Psi=1 / \sqrt{2}(\boldsymbol{g} \boldsymbol{u}-\boldsymbol{u g})=1 / \sqrt{2}(\boldsymbol{f} \boldsymbol{b}-\boldsymbol{b} \boldsymbol{f})=1 / \sqrt{2}(\boldsymbol{l} \boldsymbol{r} \boldsymbol{r} \boldsymbol{l})
$$

where $l$ and $r$ indicate the left and right position of the emitter site, in the laboratory system, respectively. This situation may be compared most closely to the Walborn experiment ${ }^{20,21}$ without selection of any component of the two $\pm 45^{\circ}$ polarizers for circular polarization behind the double slit. However, there is no tunnelling between the two slit sites. Hence, the rotating linear polarizer in the double-slit free path of the entangled photon beam projects the 'which way' information on the screen, showing fringes, left slit projections, antifringes and right slit projections. To reveal this inherent asymmetry in our case requires a second electron to be removed on a time scale similar or smaller than the tunnelling time as a "which way" marker in a reference system defined by the detection direction of the reference electron. In practice this would be a subsequent Auger electron with $\Gamma_{\mathrm{A}}<\Delta \mathrm{E}_{\mathrm{gu}}$ causing partial degeneracy of the symmetry states through lifetime broadening. This gives rise to a non-vanishing interference term $\pm 2 g u$ and hence to $f / b$ asymmetries. This degenerate $f / b$ system is an entangled state regarding the continuous variable position (see equation 2). In contrast to entanglement of discrete variables, position already displays interference behaviour on the level of the symmetry basis states. Their character as nonlocal spatial superpositions ${ }^{14}$ is clearly exhibited as oscillation in momentum space (see Fig.4).

Both the two- and the one-centre coherence between scattered and unscattered electrons can be unambiguously distinguished by their characteristic frequencies in momentum space, which is a single fixed frequency for coherent two-centre superposition, but is angle-dependent for localized one-centre self-interference. This can be clearly seen in figure 5 for a variety of angles for $\mathrm{N}_{2}$ and CO. Asymptotically, the EXAFS-oscillation disappears at shorter wavelength due to the decrease of scattering probability with increasing photoelectron kinetic energy. Hence, the dominant spatial coherence pattern of the undisturbed emitter, which reflects the core hole tunnelling, is the only coherence-driven oscillation surviving in photoelectron emission at high kinetic energies ${ }^{37,38}$. In this limit, the amplitudes of the $g$ and $u$ oscillations are the same.

\section{SUMMARY}

We have presented evidence for a scattering-induced scaledependent transition of quantum coherence in molecular coreelectron photoionization. The observed effect is manifested as an oscillation with an angle-dependent frequency in momentum space that is caused by the self-interference of photoelectrons emitted from one atomic site only, the fingerprint of localization. 
However, this effect conserves the symmetry of the two emitter sites but without spatial phase-coupling due to local separation of the emitted electron. This scenario is the result of ongoing tunnelling after collapse of the wavefunction in real space. It displays a loss of coherence along with a subtle relationship between three time domains, i. e., the scattering induced localization, the tunnelling, and the core-hole life-time. The observed effects in the photoelectron diffraction of $\mathrm{N}_{2}$ might not only contribute to a deeper understanding of the transition between the classical and the quantum world ${ }^{2,3}$, as decoherence theory, but also to more actual problems as the occurrence of single particle nonlocality ${ }^{14}$.

\section{METHODS}

The semi-classical model function $f_{\text {model }}$, employed here to describe the states $f_{s}$ and $b_{s}$ of the scattered electron, is the sum of two damped sine functions $f_{2 \mathrm{c}}^{\mathrm{T}, \mathrm{damp}}$ and $f_{1 \mathrm{c}}{ }^{\mathrm{T}, \mathrm{damp}}$, where the subscripts $1 \mathrm{c}$ and $2 \mathrm{c}$ denote 1 - and 2-centre scattering coherence, respectively. The transition function $T_{2 \mathrm{c}}(\mathrm{k})$ shown in Fig $5 \mathrm{~b}$ is chosen in analogy to the decoherence curves of Ref. 8 and Ref. 9 whereas $T_{\mathrm{lc}}(\mathrm{k})$ describes the onset of the 1-centre scattering:

$$
\begin{aligned}
& f_{\text {model }}(k)=f_{2 \mathrm{c}}^{\mathrm{T}, \text { damp }}+f_{1 \mathrm{c}}^{\mathrm{T}, \text { damp }} \\
& f_{2 \mathrm{c}}^{\mathrm{T}, \mathrm{damp}}(k)=T_{2 \mathrm{c}}(k) \cdot f_{2 \mathrm{c}}^{\mathrm{damp}}(k), \\
& f_{1 \mathrm{c}}^{\mathrm{T}, \mathrm{damp}}(k)=T_{1 \mathrm{c}}(k) \cdot f_{1 \mathrm{c}}^{\text {damp }}(k) \\
& T_{2 \mathrm{c}}(k)=\frac{1}{1+e^{\left(k-k_{2 \mathrm{c}}\right) / \Gamma_{2 \mathrm{c}}}}, \\
& T_{1 \mathrm{c}}(k)=\frac{1}{1+e^{-\left(k-k_{1 \mathrm{c}}\right) / \Gamma_{1 \mathrm{c}}}}, \\
& f_{2 \mathrm{c}}^{\text {damp }}(k)=e^{-\tau_{0} k}\left(A_{2 \mathrm{c}} \cdot \sin \left[k \cdot\left(R-r_{s}\right)+\varphi_{2 \mathrm{c}}\right]\right), \\
& f_{1 \mathrm{c}}^{\text {damp }}(k)=e^{-\tau_{0} k}\left(A_{1 \mathrm{c}} \cdot \sin \left[k \cdot\left(2 R-r_{s}\right)+\varphi_{1 \mathrm{c}}\right]\right)
\end{aligned}
$$

Here, $R$ denotes the internuclear distance or bond length and $k r_{s}$ and $\varphi_{1 \mathrm{c}, 2 \mathrm{c}}$ the energy-dependent and energy-independent phase shifts, respectively. $\tau_{0}$ is an empirical parameter describing the declining scattering intensity. In the text we referred to this combined one- and two-centre coherence model of the scattered electron as Generalized Cohen-Fano (GCF) model.

The RCHF code treats the symmetry basis states $g$ and $u$ as pure states as defined in (1a). The Cohen-Fano oscillation is the result of the interference terms $\pm 2 f b$. The EXAFS oscillation however, is an independent modulation of the intensity of the two symmetry channels $g$ and $u$. It can be described by non-interfering $f_{s}$ and $b_{s}$ scattering channels which appear with the same strength in the still non-interfering symmetry channels $g$ and $u$.

\section{Received 7 November 2007; accepted 9 May 2008; published XX Month XXXX.28}

\section{References:}

1. Jönsson, C. Elektroneninterferenzen an mehreren künstlich hergestellten Feinspalten. Z Phys. 161, 454-474 (1961)

2. Zurek, H. W., Decoherence and the transition from quantum to classical, Physics Today, October, 3644 (1991).

3. Tegmark, M. Apparent wave function collapse caused by scattering, Found. Phys. Lett. 6, 571-590, (1993).

4. Rauch, H. \& Summhammer, J. Static versus time-dependent absorption in neutron interferometry. Phys. Lett. 104A, 44-46 (1984)

5. Mittelstaedt, P., Prieur, A. \& Schieder, R. Unsharp Particle-Wave Duality in a Photon Split-Beam Experiment. Found. Phys. 17, 891-903 (1987).

6. De Martini, F., De Dominicis, L., Cioccolanti, V. \& Milani, G. Stochastic interferometer. Phys. Rev. A 45, 5144-5153 (1992).

7. Dürr, S., Nonn, T. \& Rempe, G. Origin of quantum-mechanical complementarity probed by a 'whichway' experiment in an atom interferometer. Nature 395, 33-37 (1998).

8. Kokorowski, D.A., Cronin, A.D., Roberts, T. D. \& Prichard, D.E. From Single-to Multiple-Photon Decoherence in an Atom Interferometer. Phys. Rev. Lett. 86, 2191-2195 (2001).

9. Hackermüller, L., Hornberger, K., Brezger, B., Zeilinger, A. \& Arndt, M. Decoherence of matter wave by thermal emission of radiation. Nature 427, 711-714 (2004)
10. Cohen, H. D. \& Fano, U. Interference in the Photo-Ionization of Molecules. Phys. Rev. 150, 30 -33(1966).

11. Englert, B.-G. Fringe Visibilty and Which-Way Information: An Inequality. Phys. Rev. Lett. 77, 2154 2157 (1996)

12. Uiberacker, M. et al. Attosecond real-time observation of electron tunnelling in atoms, Nature 446, 627-632 (2007).

13. Pavlychev, A. A. et al. Dynamic properties of $\mathrm{N}$ and $\mathrm{O} 1 \mathrm{~s}^{-1} \mathrm{\sigma}_{u}{ }^{*}$ shape resonances in $\mathrm{N}_{2}$ and $\mathrm{CO}_{2}$ molecules. Phys. Rev. Lett. 81, 3623-3626 (1998).

14. Dunningham J. \& Vedral V. Nonlocality of a single particle, Phys. Rev. Lett. 99, 180404-1-4 (2007)

15. Aspect, A., Grangier, P. \& Roger, G. Experimental realization of Einstein-Podolsky-Rosen-Bohm Gedankenexperiment: a new violation of Bell's inequalities. Phys. Rev. Lett. 49, 91-94 (1982).

16. Rolles, D. et al. Isotope-induced partial localization of core electrons in the homonuclear molecule $\mathrm{N}_{2}$. Nature 437, 711-715 (2005).

17. D. Akoury et al. The Simplest Double Slit: Interference and Entanglement in Double Photoionization of $\mathrm{H}_{2}$, Science 318, 949 - 952 (2007).

18. Wollenhaupt, M. et al. Interferences of Ultrashort Free Electron Wave Packets. Phys. Rev. Lett. 89, 173001 (2002).

19. Lindner, F. et al. Attosecond Double-Slit Experiment. Phys. Rev. Lett. 95, 040401 (2005)

20. Walborn, S. P., Terra Cunha, M. O., Pádua, S. \& Monken, C. H. Double-slit quantum eraser. Phys. Rev. A 65, 33818 (2002)

21. Walborn, S. P., Terra Cunha, M. O., Pádua, S. \& Monken, C. H. Quantum Erasure. American Scientist 91, 336-343 (2003)

22. Liebsch, A. Theory of Angular Resolved Photoemission from Adsorbates. Phys. Rev. Lett. 32, 12031206 (1974).

23. Kevan, S. D., Rosenblatt, D. H., Denley, D., Lu, B.-C. \& Shirley, D. A. Normal Photoelectron Diffraction of the Se 3d Level in Se Overlayers on Ni (100). Phys. Rev. Lett. 41, 1565 -1568 (1978).

24. Shigemasa, E. et al. Angular Distributions of 1 so Photoelectrons from Fixed-in-Space $\mathrm{N}_{2}$ Molecules. Phys. Rev. Lett. 74, 359-362 (1995)

25. Heiser, F. et al. Demonstration of Strong Forward-Backward Asymmetry in the C1s Photoelectron Angular Distribution from Oriented CO Molecules. Phys. Rev. Lett. 79, 2435-2437 (1997).

26. Landers, A. et al. Photoelectron Diffraction Mapping: Molecules Illuminated from Within. Phys. Rev. Lett. 87, 013002 (2001).

27. Becker, U. Angle-resolved electron-electron and electron-ion coincidence spectroscopy: new tools for photoionization studies. J. Electron Spectrosc. Relat. Phenom. 112, 47-65 (2000).

28. Lucchese, R. R., Raseev, G. \& McKoy, V. Studies of differential and total photoionization cross sections of molecular nitrogen. Phys. Rev. A 25, 2572-2578 (1982).

29. Zimmermann, B., Wang, K. \& McKoy, V. Circular dichroism in K-shell ionization from fixed-in-space $\mathrm{CO}$ and $\mathrm{N}_{2}$. Phys. Rev. A 67, 042711 (2003).

30. Hergenhahn, U., Kugeler, O., Rüdel, A., Rennie, E. E. \& Bradshaw, A. M. Symmetry-Selective Observation of the $\mathrm{N}$ 1s Shape Resonance in $\mathrm{N}_{2}$. J. Phys. Chem. A 105, 5704-5708 (2001).

31. Liu X.-J. et al. Young's double slit experiment using core-level photoemission from $\mathrm{N}_{2}$ : revisiting Cohen-Fano's two centre interference phenomenon. J. Phys. B: At. Mol. Opt. Phys. 39, 4801-4817 (2006).

32. Walter, M. \& Briggs, J. Photo-double ionization of molecular hydrogen. J. Phys. B: At. Mol. Opt. Phys. 32, $2487-2501$ (1999)

33. Fojon, O. A., Fernandez, J., Palacios, A., Rivarola, R. D. \& Martin, F. Interference effects in $\mathrm{H}_{2}$ photoionization at high energies. J. Phys. B: At. Mol. Opt. Phys. 37, 3035 -3042 (2004).

34. Fernandez, J. Fojon, O., Palacios, A. \& Martin, F. Interference from fast electron emission in molecular photoionization. Phys. Rev. Lett. 94, 043005 (2007).

35. Juanes-Marcos, J. C. , Althorpe S. C. \& Wrede E., Theoretical Study of Geometric Phase Effects in the Hydrogen-Exchange Reaction, Science 309, 1227-1230 (2005)

36. Scully. M. O. Englert, W. \& Walther, H. Quantum optical test of complementarity. Nature. 351, 111116 (1991).

37. Stolterfoht, N. et al. Evidence for Interference Effects in Electron Emission from $\mathrm{H}_{2}$ Colliding with 60 $\mathrm{MeV} / \mathrm{u} \mathrm{Kr}^{34+}$ Ions. Phys. Rev. Lett. 87, 23201 (2001).

38. Misra, D., Kadhane, U., Singh, Y. P., Tribedi, L. C., Fainstein, P. D. \& Richard, P. Interference Effect in Electron Emission in Heavy Ion Collisions with $\mathrm{H}_{2}$ Detected by Comparison with the Measured Electron Spectrum from Atomic Hydrogen. Phys. Rev. Lett. 92, 153201 (2004).

Supplementary Information accompanies this paper on www.nature.com/naturephysics

\section{Note added in proof:}

The predicted coherent localized state in the complementary $f / b$ system has been experimentally confirmed by photoelectron-Auger electron coincidence experiments in the molecule frame. The corresponding paper by Schöffler, M. S. et al. "Ultrafast probing of core hole localization in $\mathrm{N}_{2}$ " appeared in Science 320, 920-923 (2008) during proof correction.

\section{Acknowledgements}

The authors acknowledge financial support by the Bundesministerium für Bildung und Forschung (BMBF Contract No. 05KS4EB1/3). B. Z. and D. R. thank the Alexander von Humboldt Foundation for a Feodor Lynen fellowship. The authors are grateful to David Shirley for providing initial beam time for this project at the Advanced Light Source. They also thank Eddy Moler for his help during this initial period, Georg Prümper for his contribution to the development and implementation of the detector, and Danielle Dowek and Amulya Roy for helpful comments and critical reading of the manuscript.

Correspondence and requests for material should be addressed to U.B.

Supplemntary Information accompanies this paper on www.nature.com/naturephysics

\section{Author contributions}

The calculations were performed by BZ and VM whereas the measurements and the analysis of the experimental data have been done by the other authors.

\section{Author information}

Reprints and permission information is available at httpt://npg.nature.com/reprintsandpermissions. Correspondence and requests for materials should be addressed to U.B. 


\section{SUPPLEMENTAL INFORMATION}

\section{EXAFS Self-Interference and the ocurence of shape resonances}

For emission out of the molecule along the molecular axis, the direct wave interferes with an electron wave that is scattered an odd number of times and dominated by single-scattering $(66 \%$ back-scattering), whereas for emission into the molecule, the direct wave interferes with an electron wave scattered an even number of times. This process is dominated by double scattering $(34 \%$ forward scattering), as depicted in the inset of Fig. 2. The frequency of the resulting oscillation depends on the observation angle and, for observation along the molecular axis, is proportional to twice the internuclear distance (bond length $R$ ) in both cases, because the path length differences between the emitter reference atom are the same, as shown in the inset of Fig. 2. However, the apparent difference in path length is only approximately equal to the actual path-length difference of twice the bond length due to the potential-dependent scattering-induced phase shift ${ }^{1}$ in the expression for Extended X-Ray Absorption Fine Structure (EXAFS) oscillations (see methods). In our case the Fourier transform yields an apparent path length of $1.73 R$ instead of $2 R$ for the dominant backward channel, corresponding to an energydependent phase shift $k r_{\mathrm{s}}$ with $r_{s}=0.28 \AA$ and an energy independent one of $\varphi=1.3 \pi$. These values are in good agreement with predictions of potential scattering of the different partial waves. In the following, the term "double" bond length always refers to this effective double bond length. The scattering problem we are dealing with here is the one-dimensional equivalent of the EXAFS situation observed in X-ray absorption spectra ${ }^{2}$. The unequal oscillation amplitude in the backward and forward channels reflects the intrinsic asymmetry of a one-dimensional system such as a heteronuclear diatomic molecule for emission into and out of the system. Interchanging the emitter sites just reverses the meaning of forward and backward. This feature gives rise to the mirror symmetric molecular frame angular distributions at the two Kedges.

Note that at first glance the well-known shape resonance in $\mathrm{K}$ shell photoionization of $\mathrm{CO}^{3}$ is seen in the forward channel only. Its main contribution is four-fold scattering of the photoelectron oscillating with twice the frequency of the characteristic doublescattering oscillation in this channel. In addition, a double- and single-scattering contribution to an energetically-shifted shape resonance appears in both the forward and backward channels, but is masked by the close-lying stronger EXAFS scattering oscillation amplitude at higher energy. There is a transition region between the two regimes of interferometric EXAFS coherence for $\lambda$ smaller than $R$ on the one hand, and the standing waves supporting Near-Edge X-Ray Absorption Fine Structure coherence (NEXAFS) for $\lambda$ larger than $R$ on the other hand ${ }^{4-6}$. The different contributions of the two shape resonances at energies corresponding approximately to de Broglie wavelengths of two and four times the apparent bond length in the forward and backward channel are marked by bars in Fig. 2. These features are mostly unexplored at present and will not be further discussed here.

The sum of the contributions for the two channels exhibits an oscillation dominated by the backward channel with its stronger oscillation amplitude due to the larger probability for single scattering compared to double scattering. To make this effect more evident, the diffraction intensities have been normalized to the partial cross sections to eliminate its exponential decay behaviour and to display the oscillatory structure of the diffraction intensity more clearly. The agreement between this superposition and $a b$ initio curves from threshold to the high energy limit shows that this simple one-centre interference model is not only capable of describing the EXAFS region properly, but the more subtle NEXAFS regime ${ }^{7}$, as well.
In addition to the self-interference structure, the unmodulated asymptotic limit of $\mathrm{CO}$ corresponds to a macroscopic double-slit experiment with one slit open while the other one is closed. This is in contrast to the homonuclear case where the spatial coherence between the two emitter sites and its associated interference pattern is expected to dominate the corresponding asymptotic behaviour. The different scattering contributions were separated out by a fitting procedure based on the generalized Cohen-Fano (GCF) model augmented by a standing-wave description of the shape resonances.

Experimentally, for two data points at low energy, the combined photon energy and electron spectrometer resolution was sufficiently high to allow separation of the $g$ and $u$ states by a parametric fit of the line structure. The diffraction intensities at higher energies had to be derived by fixing all parameters except the line intensities. For energies with markedly different $g$ and $u$ intensities, the results of this procedure could be independently confirmed by analysis of the corresponding symmetry-sensitive fragment ion angular distributions.

The coherence transition seen in the ungerade channel is also present in the gerade channel, except that a prominent multiple scattering shape resonance appears in the two-centre coherence regime. Careful analysis of the structural appearance of this resonance shows that its origin is triple- and four-fold scattering corresponding to a path length difference of four bond lengths. Therefore, the resonance at the de Broglie wavelength with this difference in path length gives rise to a particularly pronounced NEXAFS-like superposition because, for $\lambda / 4=R$, a standing wave accompanied by electron trapping that is sensitive to the bond length ${ }^{6-8}$ can be supported. The odd and even components of the reflected wave are transferred into the forward/backward channels of the localized heteronuclear diffraction intensities and give rise to pronounced oscillations at low kinetic energies. The corresponding results for $\mathrm{CO}$ suggest that the fourfold-scattering shape resonance appears roughly as a single-period oscillation of the scattering intensity with a frequency proportional to four times the bond length. Except for the occurrence of multiple scattering in the gerade channel, the coherence transition is completely analogous to that of the ungerade channel.

One consequence of the equivalence of the sum of the amplitudes in both the $g / u$ and $f / b$ systems is that beyond the effective EXAFS threshold, i.e., for a matter wavelength $\lambda$ smaller than $R$, the scattering behaviour in the symmetric $g / u$ system must have EXAFS-like character because this is the exclusive superposition mode on the non-oscillating background in the asymmetric $f / b$ system of CO. Coexistence between the two modes of one- and two-centre coherence of scattering behaviour occurs in the predominantly standing-wave or shape resonance regime above the de Broglie wavelength $\lambda=R$ only. This argument for the observed effect on the basis of completeness of the two-dimensional Hilbert space of a one-dimensional system is complementary to that based on the more familiar uncertainty principle. The parity eigenstates $g$ and $u$ and their counter parts, the momentum eigenstates $f$ and $b$ constitute two two-dimensional Hilbert spaces analogous to the CP eigenstates $K_{s}$ and $K_{l}$ and their counterparts $K$ and $\bar{K}$ of the neutral Kaon system. The latter one are eigenstates of the discrete flavour quantum number strangeness \pm 1 in the standard model. They show a time-dependent oscillation as fingerprint of their entanglement ${ }^{9}$.

\footnotetext{
Supplemental References

1. Woodruff, D. P. et al. Direct methods in photoelectron diffraction; experiences and lessons learnt based on the use of the projection method. J. Phys. Condens. Matter 13, 10625-10645 (2001).

Sayers, D. E. \& Stern, E. A. New Technique for Investigating Nanocrystalline Structures: Fourier
} Analysis of the Extended X-Ray-Absorption Fine Structure. Phys. Rev. Lett. 27, 1204-1207 (1971). 
3. Dehmer, J. L. \& Dill, D. Shape Resonances in K-Shell Photoionization of Diatomic Molecules. Phys. Rev. Lett. 35, 213-215 (1975).

4. Rescigno, T. N. \& Langhoff, P. W. K-Shell Photoionization in Molecular Nitrogen. Chem. Phys. Lett. 51, 65-70 (1977).

5. Bianconi, A., Peterson, H., Brown, F. C. \& Bachrach, R. Z. K-shell photoabsorption spectra of $\mathrm{N}_{2}$ and $\mathrm{N}_{2} \mathrm{O}$ using synchrotron radiation. Phys. Rev. A 17, 1907-1911 (1978).

6. Stöhr, J. et al. Bonding and Bond Lengths of Chemisorbed Molecules from Near-Edge X-RayAbsorption Fine-Structure Studies. Phys. Rev. Lett. 51, 2414-2417 (1983).

7. Stöhr, J. NEXAFS Spectroscopy. Springer Series in Surface Sciences, ed. by Ertl, G., Gomer, R. \& Mills, D.L. (Springer-Verlag Berlin Heidelberg New York, 1992).

8. Thomas, T. D. et al. Photon Energy Dependence of the $1 \sigma_{\mathrm{u}} / 1 \sigma_{\mathrm{g}}$ Intensity Ratio in Carbon $1 \mathrm{~s}$ Photoelectron Spectroscopy of Ethyne. Phys. Rev. Lett. 82, 1120-1123 (1999).

9. Afriat, A. Kaons and Bell's Inequality, in The Foundations of Quantum Mechanics, edited by Garola C. \& Rossi A. p. $19-30$ (World Scientific, Singapore, 2000). 